\title{
Inverted-U effects generalize to the judgment of subjective properties of faces
}

\author{
Alain Mignault \\ McGill University, Montreal, Quebec, Canada \\ A. A. J. Marley \\ University of Victoria, Victoria, British Columbia, Canada \\ AND \\ Avi Chaudhuri \\ McGill University, Montreal, Quebec, Canada
}

\begin{abstract}
Researchers studying absolute identification have long known that it takes more time to identify a stimulus in the middle of a range than one at the extremes. That is, there is an inverted- $U$ relation between mean response time and response position. In this task, an inverted-U relation also exists between response uncertainty and response position. Similarly, an inverted-U relation between mean response time and response position has been found for psychometric measures involving questions about the self. However, psychophysicists explain these inverted-U effects differently than do self-schema researchers. We propose an integrative framework in which task constraints explain these effects. To verify the generality of these inverted-U effects, we hypothesized that they would exist in three tasks having similar constraints - in this case, tasks involving the judgment of subjective properties of faces on a Likert-type scale. Our results are consistent with this hypothesis. We discuss the relevance of the results for other applications of Likert-type scales.
\end{abstract}

Researchers from several disciplines have found that participants make judgments more quickly at the extremes of a set of ordered response categories than in the middle. In other words, there is an inverted- $U$ relation between mean response times (RTs) and response position. This inverted-U RT effect has been identified in domains as diverse as absolute identification (Brown, Marley, Donkin, \& Heathcote, 2008; Monahan \& Lockhead, 1977) and psychometric tests (Akrami, Hedlund, \& Ekehammar, 2007; Casey \& Tryon, 2001; Catanzaro, 1997; Crandall, 1998). In an absolute identification task, each participant makes judgments of a physical property of stimuli, such as the length of a bar. The inverted-U RT effect describes the fact that participants take less time to judge the shortest and longest bars of the range. In the psychometric tests in which the inverted-U RT effect has been found, participants are typically asked to evaluate how much they agree with a given statement about themselves, using a Likert-type scale. In this context, the inverted-U RT effect describes the fact that participants respond faster when they strongly agree or strongly disagree with a statement. In absolute identification, researchers have also found a related effect, that the diversity of responses provided for a stimulus, or response uncertainty, is less for stimuli at the extremes than for those in the middle of the presented range (Avant, Bevan, \& Wing, 1968; Behar, 1963). We call this effect an inverted-U response uncertainty effect. Similarly, in the field of psychometrics, many researchers have remarked that there is greater ambiguity in the middle of Likert-type scales than at the extremes (e.g., Coolican, 1994; Weems \& Onwuegbuzie, 2001).

Psychophysicists (Brown et al., 2008; Lacouture \& Marley, 1995; Monahan \& Lockhead, 1977; Stewart, Brown, \& Chater, 2005) explain these inverted-U effects differently than do self-schema researchers (Akrami et al., 2007; Casey \& Tryon, 2001; Catanzaro, 1997; Crandall, 1998; Kuiper, 1981). Self-schema researchers usually explain them by a two-process mechanism whereby judgments about the self activate a process different from that involved in the judgment of unfamiliar others. Psychophysicists attribute the inverted- $U$ effects to stimulus representation and response selection processes. Here, we adopt the psychophysical perspective by testing an integrative framework in which inverted-U effects are part of a general phenomenon that arises in any task of judging stimuli on a response scale involving polytomous (i.e., more than two) ordered categories.

As detailed in the next section, every successful contemporary model of choice in unidimensional absolute identification - and, when included, RT — involves a unidi-

A. Mignault, alain@ego.psych.mcgill.ca 
mensional representation of the relevant stimulus attribute, followed by some form of limited capacity mapping of that stimulus representation to a response selection phase. The limited capacity has been motivated in various ways: as noise in the mapping (e.g., Lacouture \& Marley, 1995); as a stimulus-based selective attention process (e.g., Brown et al., 2008; Marley \& Cook, 1984); or as noise in the response selection process (Stewart et al., 2005). The task also involves the important constraint of mapping a unidimensional stimulus representation to a vector of evidence values, one for each possible response (e.g., Lacouture \& Marley, 1995). Because the unidimensional representation and the noise lead to a limited capacity, and because the response selection mechanism involves constraints, we call our proposal the limited capacity with selection constraints hypothesis. We propose that similar processes are involved whether the participant is judging objective or subjective properties of stimuli, or answering questions about the self, others, or the world on an attitude scale. The originality of this hypothesis lies not in the mechanisms proposed but in the generalization from absolute identification to subjective judgments of a variety of stimuli (e.g., faces, questions about the self and others) on a response scale of polytomous ordered categories.

To show the generality of the inverted-U effects that arise from this approach, we tested whether they exist in a task with similar constraints for which they had not been found before - namely, the judgment of subjective properties of faces on a Likert-type scale. Our results are consistent with the hypothesis. To contextualize our findings, we review the relevant literature regarding response uncertainty, absolute identification, and Likert-type scaling.

\section{Dependent Variables}

To test whether the inverted-U effects found in absolute identification generalize to the judgment of subjective properties of stimuli, we asked participants to rate faces on Likert-type scales. Each scale that we used represents a subjective property of the stimuli, such as the similarity between two faces. In other words, as opposed to absolute identification, there is no objective criterion on which to evaluate the answer. This means that there are no right or wrong answers, and one cannot compute an accuracy score, an error rate, or a $d^{\prime}$. Therefore, we could not study the inverted-U error effect and instead focused on measuring the other two inverted- $U$ effects found in absolute identification: the inverted-U RT effect and the inverted-U response uncertainty effect. In this article, both mean RT and response uncertainty are computed across participants for a given stimulus. Response uncertainty measures the diversity of responses for a specific stimulus, using Stilson, Haaseth, Walsmith, Schneider, and Rogers's (1972) equation of mean conditional response uncertainty:

$$
H\left(R \mid S_{j}\right)=-\sum_{i=1}^{N} P\left(R_{i} \mid S_{j}\right) \log _{2} P\left(R_{i} \mid S_{j}\right),
$$

where $H\left(R \mid S_{j}\right)$ is the conditional response uncertainty associated with the $j$ th stimulus $S_{j}, P\left(R_{i} \mid S_{j}\right)$ is the probabil- ity that the $i$ th response $R_{i}$ will be given when stimulus $S_{j}$ is presented, and $N$ is the number of response choices. In much of the literature and in this article, $P\left(R_{i} \mid S_{j}\right)$ are estimated from response frequencies across participants. The variable $j$ takes the values from 1 to $m$, where $m$ is the number of stimuli. To simplify, throughout the article we will use the expression "response uncertainty" instead of "mean conditional response uncertainty." Response uncertainty is the amount of information in the response, measured in bits (binary digits; Shannon \& Weaver, 1964). Response uncertainty is maximal when the response probabilities $P\left(R_{i} \mid S_{j}\right)$ all equal $1 / N$ (Shannon \& Weaver, 1964) and minimal (zero) when a particular response $R_{k}$ is certain to occur [i.e., when $P\left(R_{k} \mid S_{j}\right)=1$ and $P\left(R_{i} \mid S_{j}\right)=0$ for $1 \leq i, k \leq N$, and $\left.i \neq k\right]$. For example, when there are three response choices $(N=3)$, if the response probabilities for a given stimulus were 1,0 , and 0 , then the response uncertainty for that stimulus is 0 bits (i.e., the minimum), whereas if the response probabilities were $.33, .33$, and .33 , then the response uncertainty is 1.585 bits (i.e., the maximum). To summarize, when response frequencies are spread over the available choices, response uncertainty is high.

\section{Absolute Identification}

Three inverted-U effects have been found in absolute identification (for reviews, see Luce \& Nosofsky, 1984; Miller, 1956; Shiffrin \& Nosofsky, 1994; Stewart et al., 2005). First, there is an inverted-U relation between error rate and stimulus rank (Braida \& Durlach, 1972; Marley \& Cook, 1984). This inverted-U relation is also called a bow effect, edge effect, end-anchor effect, or serial position effect. We prefer the term inverted-U error effect because the expression "inverted-U" is more often used in other fields. Second, there is an inverted- $U$ relation between response uncertainty and stimulus rank (Avant et al., 1968; Behar, 1963), illustrated by the solid line in Figure 1 for Lacouture and Marley's (1995) data. Third, there is an inverted- $\mathrm{U}$ relation between mean RT and stimulus rank (Lacouture, Li, \& Marley, 1998; Lacouture \& Marley, 1995, 2004), which has also been found in the absolute identification of bidimensional stimuli (Monahan \& Lockhead, 1977).

Inverted-U effects in absolute identification display a number of other characteristics, of which we describe two (for full details of others, see Brown et al., 2008; Stewart et al., 2005). First, the inverted-U-shaped curve for RT is progressively deeper for larger numbers of stimuli. In other words, there is a greater difference in mean RT between the middle of the response range and the ends of it when there are more stimuli (and, therefore, more responses). Second, there are sequential effects - that is, when a stimulus is repeated on consecutive trials, accuracy increases and RT decreases for that stimulus. This effect extends, but in reduced magnitude, to stimuli that are similar to the previous stimuli, leading to a "double bow" inverted-U M-shaped curve for RT when RT is plotted against the stimulus difference on consecutive trials. 


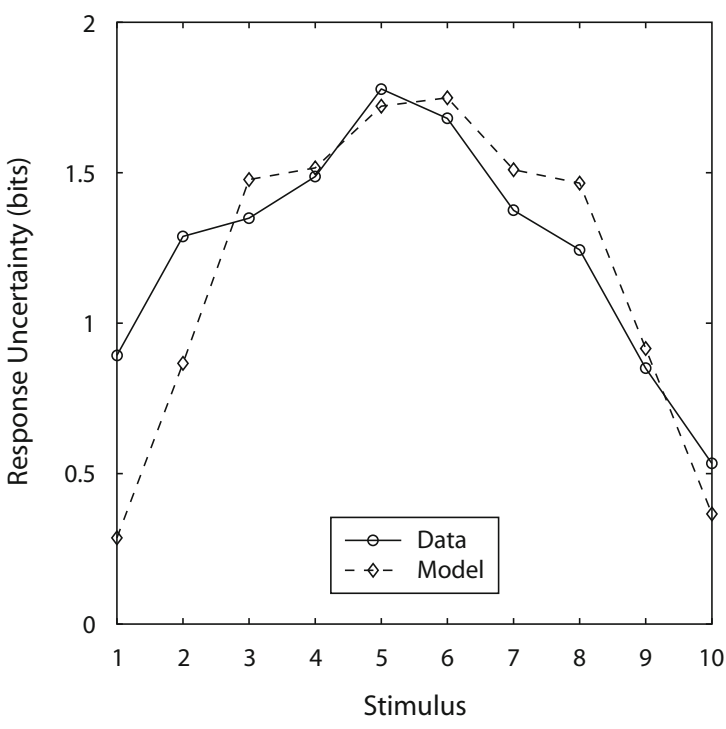

Figure 1. Inverted-U response uncertainty effects computed from Lacouture and Marley's (1995) simulation and behavioral data for the absolute identification of 10 line lengths. Data are averaged over 3 participants, with a total of 150 observations per point.

Several models of absolute identification have been proposed to predict the inverted-U RT effect and its characteristics. However, the models' predictions for the inverted-U response uncertainty effect had not previously been examined. Using Lacouture and Marley's (1995) predicted response frequencies, we computed response uncertainty and found that their model correctly predicts the inverted-U response uncertainty effect. The predictions of their model are represented by the broken line in Figure 1.

We now summarize the common aspects of three of the major models of absolute identification that predict the above inverted-U effects. We discuss Lacouture and Marley's $(1995,2004)$ original and revised noisy mapping model and Brown et al.'s (2008) selective attention, mapping, ballistic accumulation (SAMBA) model. Each of these models has the following phases: (1) stimulus representation; (2) stimulus representation to response mapping; (3) response selection; and (4) response production (i.e., motor output). The final stage is usually not modeled in detail, but is assumed to be error free and to take a constant time for each possible response; later, we give evidence that the latter assumptions are reasonable approximations.

All of these models assume that each stimulus is represented over trials at the psychophysical level by a unidimensional normal distribution with a mean determined by the stimulus and a variance that is the same for each stimulus. This assumption parallels that of Thurstone's law of categorical judgment (described in Torgerson, 1958) and of signal detection theory (Green \& Swets, 1966). However, these two earlier theories do not involve an RT mechanism; and, as the following material shows, it is not a simple matter to extend a theory (including the two just mentioned) to the accurate prediction of mean (or distributions of) RTs. In particular, in the case of absolute identification, there has to be a mechanism that maps a scalar stimulus value to a vector of response strengths, one strength for each of the possible responses in the experiment. Nonetheless, it is important to note that if one assumes an unequal variance model, then it is considerably easier to fit inverted-U effects in absolute identification; Nosofsky (1997) presents such a model. However, now it is generally accepted that making such an assumption bypasses the major conceptual issues in modeling absolute identification performance, which is to explain why such a variance depends on contextual factors such as stimulus position, number of stimuli, stimulus range, and stimulus history.

The Lacouture and Marley $(1995,2004)$ and Brown et al. (2008) models differ after the stimulus representation stage. Lacouture and Marley $(1995,2004)$ assume a simple transform of the presented stimulus to a noisy value in the range $[0,1]$, whereas Brown et al. develop a more complex attention model that also yields a noisy value in the range $[0,1]$, but with this transform dependent on the history of previous stimuli. This allows the SAMBA model to deal with certain sequential effects in a systematic fashion.

The mapping process extracts from a stimulus representation the evidence supporting each response. The stimulus-response evidence mapping has the same form in all models discussed here and can be interpreted as a connectionist model with one input unit, one hidden unit, and $N$ output units (where $N$ is the number of stimulusresponse pairs in the experiment). In this case, the hidden unit carries the psychological representation of the stimulus. This family of linear maps transforms the representation of the currently presented stimulus to $N$ outputs in such a way that, on average, the largest output is associated with the correct response for the current stimulus. Each of the three models interprets the activation of an output as the amount of evidence in favor of the corresponding response. Lacouture and Marley's $(1995,2004)$ models have an additional noise parameter at this stage; SAMBA does not. Panels D and E of Figure 2 illustrate the mapping when there are four choices. The bold lines show that, in this case, the choice with the most evidence in the $[0, .25]$ range is Choice 1 ; in the $[.25, .5]$ range, Choice 2 ; in the $[.5, .75]$ range, Choice 3 ; and in the $[.75,1]$ range, Choice 4.

The response selection mechanism in each model is based on an evidence accrual process (also called a sequential sampling process). The evidence for each response is assigned to a separate decision accumulator and accumulated within a trial until one of the accumulators reaches a predetermined state, at which time a response is made (Rouder, 2001). Lacouture and Marley's (1995) accumulator model is linear and includes neither decay of information over time nor inhibitory interactions between the accumulators, whereas the extension in Lacouture and Marley (2004) is nonlinear and includes both of the latter properties. SAMBA's (Brown et al., 2008) accumulators include only inhibition. Partly as a consequence of these 


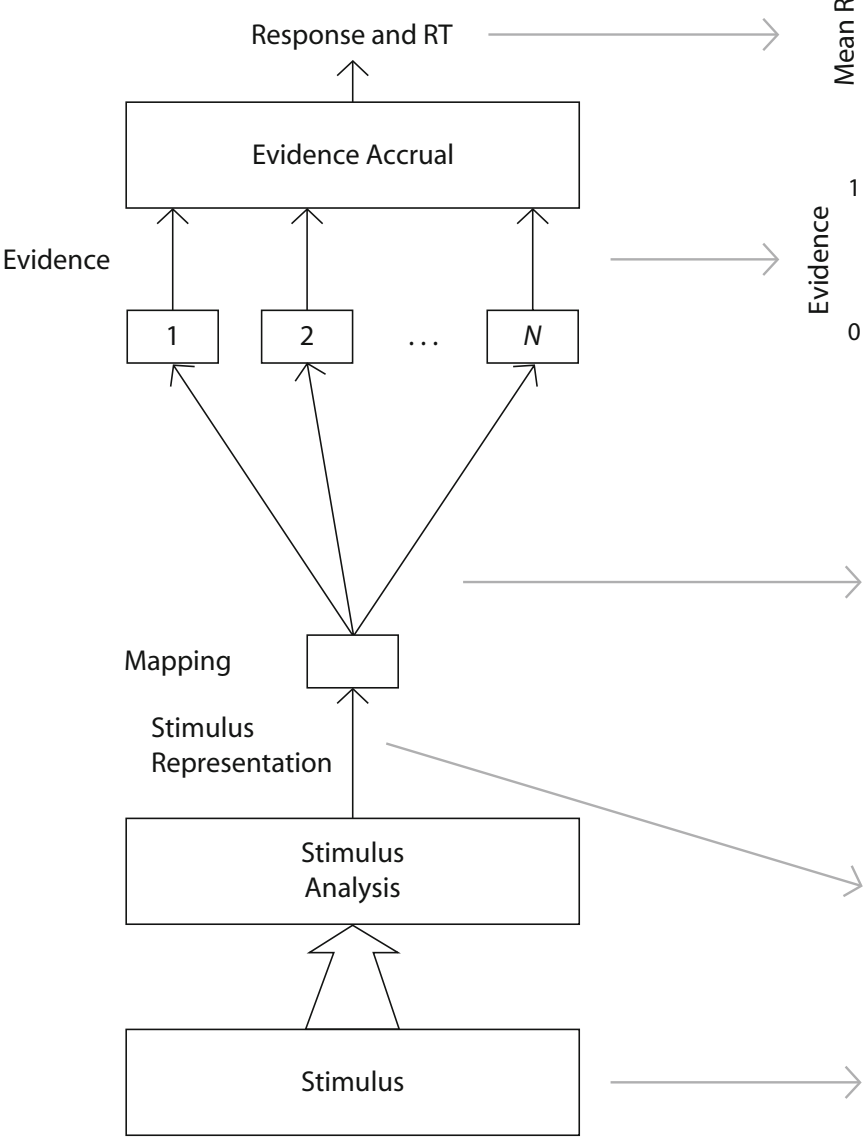

(A)
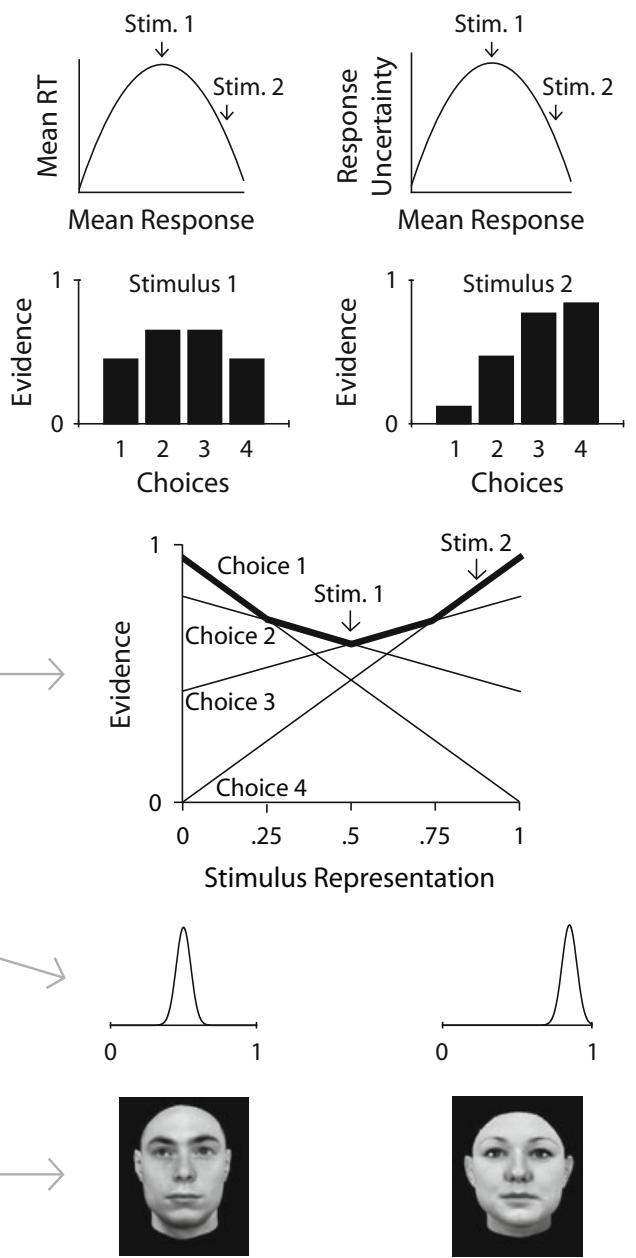

Stimulus 1
(E)

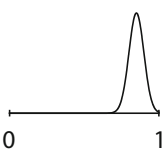

(c)

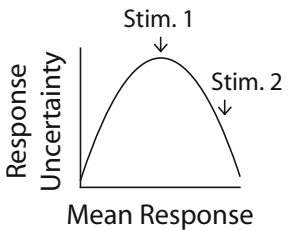

(F)

(D)

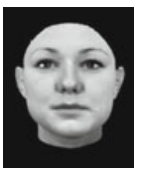

Stimulus 2

Figure 2. The limited capacity with selection constraints hypothesis. Panel A illustrates the general structure of our framework, to be read from bottom to top. Panel B illustrates two stimuli: The mean representation of Stimulus 1 is in the middle of the range, whereas for Stimulus 2 the mean is near the upper end of the range. Panel $C$ shows the unidimensional normal stimulus representations of the two stimuli in the psychological space. Panel D shows the mapping from a stimulus representation to the amount of evidence in favor of each choice, assuming a four-level Likert-type scale. Panel $E$ shows the amount of evidence (preaccrual) in favor of each choice for both stimuli. Panel F shows the inverted-U effects. This figure illustrates two trials of the gender task.

differences, a decision is reached in Lacouture and Marley's (2004) model once only one accumulator is still active (a winner-take-all mechanism), whereas in Lacouture and Marley's (1995) model and SAMBA a decision is made when an accumulator reaches a predetermined threshold.

Finally, it is important to note that each of the models leads to a limited capacity in a person's ability to correctly identify stimuli, and by fitting the data with appropriately selected parameters, each fits various "classic" absolute identification data. SAMBA currently has the advantage that it fits sequential effects.

\section{Likert-Type Scales}

Numerous researchers in psychometrics have also found an inverted-U RT effect in studies using personal- ity questionnaires and attitude questionnaires with Likerttype scales (Akrami et al., 2007; Andrews, 2003; Casey \& Tryon, 2001; Catanzaro, 1997; Crandall, 1998; Judd \& Kulik, 1980; Kuiper, 1981; Mueller, Thompson, \& Dugan, 1986). In particular, this effect was found for items of computerized versions of personality tests such as the Revised NEO Personality Assessment Inventory (NEO PI-R; Costa \& McCrae, 1992) and the Personality Assessment Inventory (PAI; Morey, 1991). Judd and Kulik (1980) also found this effect when their participants rated their agreement with 44 attitude statements.

The most common psychometric explanation for the inverted-U RT effect invokes the self-schema and, more precisely, Kuiper and Rogers's (1979) two-process interpretation (Akrami et al., 2007; Andrews, 2003; Casey \& 
Tryon, 2001; Catanzaro, 1997; Crandall, 1998). Kuiper and Rogers presented evidence suggesting that judgments about the self activate a process different from that involved in the judgment of unfamiliar others. According to this perspective, judgments of unknown others involve slow or effortful strategies, whereas judgments about the self involve highly organized and efficient schemas. For example, if a participant strongly agrees or disagrees with an item on abortion, according to this explanation it is because this item is relevant to the self, the self-schema is activated by the item, and therefore RT should be short. Kuiper (1981) also proposed that the inverted-U RT effect is evidence in favor of the self as a prototype. In his view, judgments at the extremes of the scale are faster for items that are more prototypical of the self. The inverted-U RT effect is a particular case of the cognitive properties of prototypes that are categorized faster than the less typical members of a category. However, Kuiper does not explain why both ends of a scale (for instance, strongly agree and strongly disagree) would be prototypical. Alternatively, Casey and Tryon (2001) suggested that "without recourse to the hypothetical construct of a self-schema, the inverted-U effect could be interpreted as resulting simply from the effect of stimulus contents ambiguity or uncertainty or response latency" (p. 529). However, they did not provide uncertainty measures to support their interpretation.

\section{The Limited Capacity With Selection Constraints Hypothesis}

Most self-schema researchers attribute the inverted-U RT effect to a two-process interpretation, with one process for self-relevant stimuli (or schema) and the other for otherrelevant stimuli (or schema); they additionally assume that stimulus analysis is faster for more self-relevant stimuli. However, researchers studying absolute identification attribute this effect to limited information capacity and response selection constraints. Instead of being independent effects found both in absolute identification and in self-schemas, we think that inverted-U effects in these fields are part of a more general phenomenon. In line with this perspective, we use the term "stimuli" to designate both sensory stimuli (such as facial images, lines, or tones) and questionnaire items (such as attitudes toward the world, the self, or others).

Thus, our hypothesis is that when participants judge stimuli on a scale of polytomous ordered categories, the task's constraints force the use of certain mental processes. These mental processes are similar whether the participant is identifying stimuli on the basis of their physical properties, judging the subjective properties of stimuli on a Likert-type scale, or answering questions about the self, others, or the world on an attitude scale. These common mental processes are the stimulus representation and response selection phases. Figure 2A illustrates our hypothesis. More precisely, we hypothesize that the conditions required to obtain inverted-U effects are the following: The stimulus representation over trials is a unidimensional, usually normal, distribution (see panels $\mathrm{B}$ and $\mathrm{C}$ of Figure 2); there is a family of linear transforms that maps the current stimulus representation to $N$ output values (where $N$ is the number of responses in the experiment) in such a way that, on average, the largest output is associated with the appropriate response for the current stimulus (see panels D and E in Figure 2); and there is an evidence accrual mechanism that determines the response and hence the RT (see Figure 2A).

The above assumptions produce a pattern of unit activations similar to those of Lacouture and Marley's (1995) and Brown et al.'s (2008) models. An example of this pattern of activation is presented in Figure 2D. The activation curves are farther apart at the ends than they are in the middle of the range, which shows that evidence is more unequally shared among choices near the ends than in the middle. Similarly, activation is higher for responses near the ends than in the middle of the range. Thus, this mapping also predicts a U-shaped activation effect for the choice with the greatest amount of evidence, or U-shaped evidence effect (represented by the bold line in Figure 2D). In other words, a stimulus in the middle of the range produces a smaller amount of evidence in favor of the appropriate response and also produces more evidence in favor of competing responses than does a stimulus near the ends (for more detailed explanations, see Brown et al., 2008, and Lacouture \& Marley, 1995).

The more uneven distribution of evidence among the choices near the ends than in the middle of the range leads to an inverted-U response uncertainty effect. Because the amount of evidence (preaccumulators) is more spread among the possible responses for a stimulus in the middle of the response range than for a stimulus at the ends, response probabilities (postaccumulators) are more spread out for stimuli near the middle, generating greater response uncertainty. A U-shaped evidence effect (preaccrual) also leads to the inverted-U RT effects (postaccrual) because the mean time to accumulate enough evidence to reach the response criterion is a monotonic function of the choice having the greatest amount of evidence (preaccumulator). This is the case whether a model uses leaking or not and whether a model uses lateral inhibition or not. Therefore, it takes more time to accumulate enough evidence to reach the response criterion in the middle of the range than it does at the ends.

One of the simplest models that can predict inverted-U effects in both choice and RT is Lacouture and Marley's (1995) connectionist model. Note that the various models of absolute identification capable of predicting the inverted-U RT effect are not equivalent, and vary in their ability to correctly predict the specific characteristics of the inverted-U effects (e.g., set size and sequential effects). Here, we propose a general framework - that is, a set of conditions to obtain inverted-U effects, rather than a specific model, because, in studying judgments of subjective properties of stimuli, we are only at the stage of showing that inverted-U effects exist rather than of describing their characteristics and modeling them. A complete model would require a different stimulus analysis mechanism for each task. This is analogous to absolute identification, where there are substantial variations across tasks for stimulus analysis because of variations in modalities 
(e.g., visual vs. auditory) and because of variations in the dimensionality of the stimuli (e.g., lines vs. pictures of faces). Likewise, there may be variations across tasks for the response production phase, depending on the response mode - that is, voice key or keypress. However, inverted-U effects depend only on the stimulus representation and stimulus selection phases.

\section{Presentation of Our Experiments}

In absolute identification, the stimuli are often unidimensional. However, in Likert-type scaling, the stimuli are usually multidimensional, though, of course, each Likert-type scale can capture only one dimension of the stimuli (such as their similarity). The human face constitutes a good example of a multidimensional stimulus because it carries information about identity, emotion, gender, maturity, age, ethnicity, and so on. We hypothesize that, even for such a multidimensional stimulus, the representation in the psychological space is mapped in some way to a unidimensional representation when a unidimensional response is required. Because of this multidimensionality and the importance of the face in human interactions, facial stimuli were used in the two experiments that we conducted to test our hypotheses. The first was a similarity task, in which participants had to judge the similarity between pairs of pictures of facial expressions of emotion using a Likert-type scale. In the second experiment, participants rated their perception of the maturity and gender of facial images on Likerttype scales. Not only does this constitute the first test of inverted-U effects in judgments of subjective properties of stimuli, this also constitutes the first test of these effects in face perception.

\section{EXPERIMENT 1 Judgments of Similarity}

Judgments of similarity/dissimilarity are very important for perception and cognition in general, because they allow the study of psychological representations of stimulus sets. For instance, the judgments of similarity between pairs of facial expressions of emotion make it possible to analyze the representation of emotions using a multidimensional space of facial expressions of emotion (for a review, see Mignault, Etcoff, \& Pentland, 2004). More specifically, emotion researchers ask participants to evaluate the similarity between pairs of facial expressions (e.g., Alvarado, 1996; Lemay, Kirouac, \& Lacouture, 1995; Ogawa \& Suzuki, 2000; Russell \& Bullock, 1986; Spencer-Smith et al., 2001). Using the multidimensional scaling (MDS) technique pioneered by Shepard (1962), these measures can be converted into distances within a geometric representation. The more dissimilar two facial expressions are, the more distant they are in that space. This geometric representation is sometimes referred to as the emotional space.

Here, our main goal is not to study the representation of facial expressions; we simply want to use the similarity task to assess the presence of inverted-U effects.
Our specific objectives, in this task, are to test whether there is an inverted- $\mathrm{U}$ relation between response uncertainty and mean similarity, and also between mean RT and mean similarity.

\section{Method}

Participants. Participants were 45 McGill University psychology students $(18 \mathrm{men})$ and ranged in age from 19 to 37 years. The mean age was 23.3 years, with a standard deviation of 4.9 years. Twenty participants received course credits for their participation, whereas the others were volunteers. All participants reported normal or corrected-to-normal vision.

Stimuli. Stimuli were taken from Ekman and Friesen's (1976) Pictures of Facial Affect (PFA) set. Seven pictures of the male poser $\mathrm{PE}$ and seven pictures of the female poser PF were selected. The seven categories represented were: anger, disgust, fear, happiness, neutral, sadness, and surprise. Each stimulus was normalized, which involved manually setting reference points on each face and automatically translating, cropping, and resizing each image with MATLAB programs. The contrast of the image was also normalized in such a way that every image had the same mean and variance in terms of light intensity. Paired stimuli showed the same poser displaying different emotions. All possible pairs using the seven emotions were formed, except pairs involving the same emotion. Thus, there were 84 stimulus pairs - that is, 2 posers $\times[(7 \times 7)-7]$. Figure 3 shows an example of a stimulus pair (happiness-neutral). On each trial, two dependent variables were measured - namely, similarity judgment and RT.

Apparatus. The experiment involved two tasks: The similarity task was preceded and followed by a number naming task. Both tasks were performed using an IBM-compatible computer in a quiet room. The experiment was programmed using the Micro Experimental Laboratory (MEL) software (Schneider, 1988). In both tasks, participants spoke their answers into a microphone. The screen was at a distance of approximately $74 \mathrm{~cm}$ from the participant.

Number naming task. There are differences in the time needed to name different digits (Damian, 2004). Therefore, we included a number naming task to make sure that the differences in mean RT for different similarity judgments were significantly larger than those found in the number naming task. This task involved the measurement of the naming times for the digits from 1 to 8 (i.e., the responses in the similarity task) before and after the similarity task. Each digit naming task involved four presentations per digit, for a

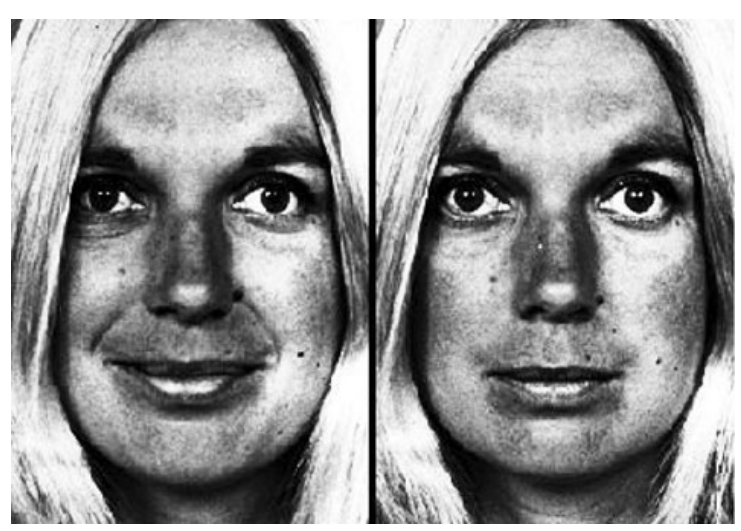

Figure 3. Example of a stimulus pair used in the similarity task. From Pictures of Facial Affect, by P. Ekman and W. V. Friesen, 1976, Palo Alto, CA: Consulting Psychologists Press. Reproduced with permission. 
total of eight presentations per digit. Order of presentation was randomized across participants and participants were instructed to name the digit as soon as it appeared on the screen. Test trials were performed at the beginning of the task, at which time the experimenter explained to the participant how to avoid inadvertently triggering the microphone by moving, making noises, or saying "hm." On each trial, the message "GET READY!" was displayed for 1,000 msec, and then a fixation cross appeared for $500 \mathrm{msec}$, after which a digit was displayed at the prior position of the cross; and the digit remained on the screen until the participant made a response or until a delay of 1,000 msec had elapsed. The computer recorded the participant's response. The RT was the time to trigger the voice key. There was a delay of 1,500 msec between trials.

Similarity task. In the similarity task, both stimuli of a pair were presented on the monitor at the same time. The average face width sustained an angle of approximately $5.8^{\circ}$. There was an angular gap of about $7.0 \mathrm{~min}$ between faces. The order of presentation of the stimulus pairs was randomized across participants. Participants were provided with 15 practice trials before the 84 real trials. Stimulus pairs were presented once. Every trial had the same structure. Each trial began with the message "GET READY!" appearing on the screen for $1,000 \mathrm{msec}$, followed by a fixation cross that was displayed for $500 \mathrm{msec}$; then, after a delay of $30 \mathrm{msec}$, the stimulus appeared until the participant made a response or until a delay of 6,000 msec had elapsed. If the delay elapsed without a response being made, an error message appeared. Participants had to evaluate the similarity between facial expressions on a scale from 1 to 8 , where 1 meant a little similar and 8 meant very similar. They were instructed to speak their judgment as soon as possible after the faces appeared on the screen. After voicing their choice, participants had to type in the number corresponding to the similarity judgment just emitted. There was a delay of $1,500 \mathrm{msec}$ between trials, after which the participant had to press a key to start the next trial.

\section{Results}

Computational considerations. The fact that there are no objective accuracy criteria when judging subjective properties of stimuli imposed two methodological differences in data analysis compared with the task of absolute identification. First, we could not use the same variable as in absolute identification for the $x$-axis - that is, the rank of a stimulus in the set of stimuli ordered according to the selected property (e.g., length). Instead, the $x$-axis corresponds to the values of the average response (e.g., similarity) for each presented stimulus. For example, assuming that for a particular stimulus $j$ the proportion of participants who responded 1,2 , or 3 is $50 \%, 30 \%$, and $20 \%$, respectively, then the average response for that stimulus would be $1.7[(.5 \times 1)+(.3 \times 2)+(.2 \times 3)]$. Second, in absolute identification, mean RT is often studied for correct responses only. With judgments of subjective properties, mean RT was computed using all responses.

Validity tests. We conducted several statistical tests to evaluate the validity of the data and to exclude outliers. In the digit naming task, the mean RTs for the numbers 1 to 8 were found to equal 446, 453, 476, 492, 478, 471, 481 , and $453 \mathrm{msec}$, respectively, with a standard error of $12 \mathrm{msec}$. These results correlate at .59 with those of Damian (2004). To evaluate measurement reliability, we used the split-sample correlation technique. This method consists of randomly dividing the group of participants into two groups, correlating the results of the two groups, repeating these steps 10 times, and averaging the results. The test-retest reliability estimate of mean RT, evalu- ated with the Spearman-Brown correction applied to this split-sample correlation, is .97. The difference between the minimum and maximum mean RT is $46 \mathrm{msec}$ (i.e., 492 - $446 \mathrm{msec}$ ). Therefore, in the similarity task, differences in mean RT greater than $70 \mathrm{msec}$ [46 msec + $2 \times(12 \mathrm{msec})]$ - for stimuli having a different mean similarity - are not likely to be caused only by differences in digit naming latencies. In the similarity task, the stimulus pairs were presented twice (in reverse order); thus, we could compute the within-participants correlation. Three participants who provided random answers (i.e., $R^{2}<.02$ ) were removed from further analyses. The average splithalf reliability estimates (computed with the SpearmanBrown correction formula) for mean similarity, response uncertainty, and mean RT equal: .956, .784, and .546, respectively. We also studied the distribution of RTs. The raw distribution of RTs was positively skewed $\left(\gamma_{1}=1.25\right.$; Snodgrass \& Yuditsky, 1996). However, computing the mean RT across participants reduced the skewness of the distribution (i.e., $\gamma_{1}=-0.45$ ) compared with the raw RTs. This degree of skewness does not adversely affect the symmetry assumption of regression (Snodgrass \& Yuditsky, 1996). Therefore, in the following pages, mean RT was untransformed.

Finally, to assess the validity of the similarity measures, we computed the emotional space of facial expressions of emotion. MDS analyses were conducted on the mean similarity judgments across both sexes using the software Multiscale II (Ramsay, 1997). The analyses involved computing a $7 \times 7$ symmetrical matrix for each participant and selecting the Diagonal option, which assumes that there is a solution common to all participants. The 2-D model was computed (Figure 4). This solution is statistically significant; that is, it is superior to the zero-dimension model $\left[\chi^{2}(135)=781, p<.000\right]$.

Inverted-U effects. In the similarity task, we expected to find an inverted-U effect in the plots of response uncertainty and of mean RT against average similarity. Figure 5

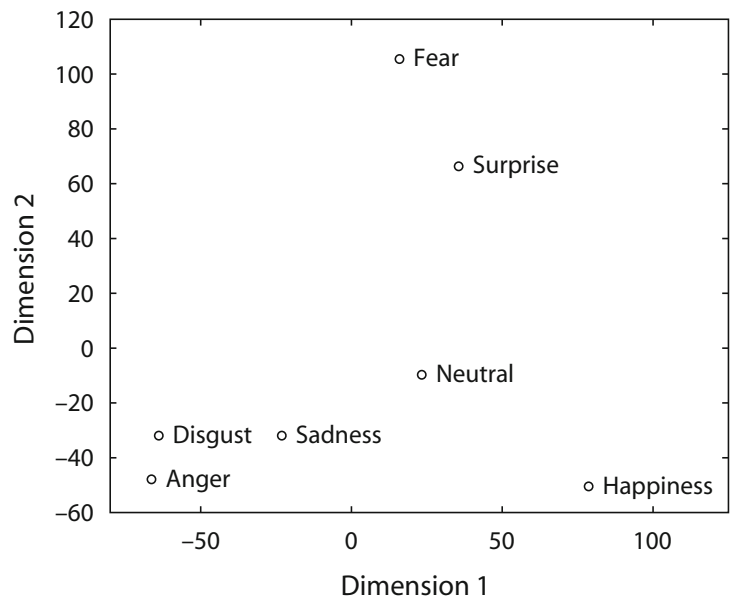

Figure 4. Two-dimensional representation of facial expressions of emotion computed with Multiscale II. 


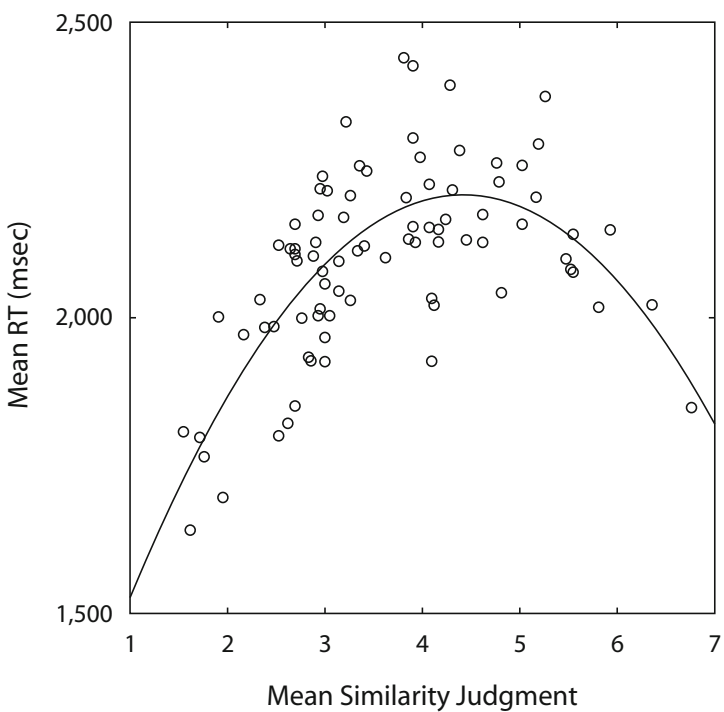

Figure 5. Mean response time (RT) as a function of mean similarity judgment for 84 stimulus pairs. Each circle represents a pair of facial expressions. The solid line represents the best-fitting quadratic equation.

plots mean RT as a function of mean similarity for each stimulus pair. In this figure, as in the following one, each circle represents a stimulus pair. There are 84 stimulus pairs on the graph and each stimulus pair is computed using 42 observations (45 participants minus 3 outliers). The graph reveals that the relation is not linear but rather quadratic, showing a maximum mean RT in the middle range of the similarity judgments. This is also demonstrated by a regression analysis. The linear equation fitting these data is significant $\left(R^{2}=.181\right)[F(1,82)=18.12$, $p<.001]$. The quadratic equation is also significant $\left(R^{2}=\right.$ $.528)[F(2,81)=45.2, p<.001]$. A partial $F$ test reveals that the quadratic equation is significantly superior to the linear equation $[F(1,81)=59.4, p<.001]$. The detailed results for each parameter of the linear and the quadratic equation are provided in Table 1. We looked at individual data by computing a mean RT for each participant on three sections of the response scale (1 to 3, 4 and 5, and 6 to 8 ). One more participant had to be excluded because no response above 4 was provided. We found that 25 out of 41 participants (i.e., 61\%) showed the inverted-U effect in RT. We also found that the average across the 16 participants who did not show an individual inverted-U RT effect showed an inverted-U mean RT effect.

The plot of response uncertainty as a function of the mean similarity judgments for each stimulus pair is shown in Figure 6. Regression analyses were performed. The best-fitting linear equation is significant $\left(R^{2}=.335\right)$ $[F(1,82)=41.35, p<.001]$. The best-fitting quadratic equation is also significant $\left(R^{2}=.912\right)[F(2,81)=421.7$, $p<.001]$. A partial $F$ test reveals that the quadratic equation is significantly superior to the linear one $[F(1,81)=533.56$, $p<.001]$. The detailed results for each parameter of the linear and the quadratic equation are provided in Table 1.

\section{Discussion}

The following characteristics are present in the emotional space that we obtained (see Figure 4): (1) Anger and disgust are very close; (2) the emotion that is the closest to fear is surprise; (3) sadness is close to both anger and disgust; and (4) happiness is farthest from the other emotions. To make the interpretation of the dimensions clearer, Figure 4 shows the representation with the coordinates rotated by $30^{\circ}$ counterclockwise relative to the solution obtained with Multiscale II. Dimension 1 accurately represents valence and Dimension 2 approximately represents arousal. These results replicate other researchers' findings (e.g., Alvarado, 1996; Lemay et al., 1995; Ogawa \& Suzuki, 2000; Russell \& Bullock, 1986; Spencer-Smith et al., 2001), thus confirming the validity of our similarity measures.

Table 1

Results of Linear and Quadratic Regressions for

(A) Response Time and (B) Response Uncertainty, As a Function of Average Similarity

\begin{tabular}{llllll}
\hline Parameter & Coefficient & Lower & Upper & & \\
Bound & Bound & $t$ & Significance \\
\hline
\end{tabular}

(A) Response Time As a Function of Average Similarity

Linear Model

$\begin{array}{crrrrr}\beta_{0} & 1,883.4 & 1,779.5 & 1,987.3 & 36.0 & .000 \\ \beta_{1} & 58.5 & 31.2 & 85.8 & 4.25 & .000 \\ \text { Quadratic Model } & & & & \\ \beta_{0} & 1,070.1 & 845.6 & 1,294.5 & 9.4 & .000 \\ \beta_{1} & 514.9 & 395.3 & 634.5 & 8.5 & .000 \\ \beta_{2} & -58.2 & -73.3 & -43.2 & -7.7 & .000\end{array}$

(B) Response Uncertainty As a Function of Average Similarity

Linear Model

\begin{tabular}{|c|c|c|c|c|c|}
\hline$\beta_{0}$ & 1.920 & 1.731 & 2.110 & 20.1 & .000 \\
\hline$\beta_{1}$ & 0.161 & 0.111 & 0.211 & 6.4 & .000 \\
\hline \multicolumn{6}{|c|}{ Quadratic Model } \\
\hline$\beta_{0}$ & -0.205 & -0.401 & -0.009 & -2.0 & .040 \\
\hline$\beta_{1}$ & 1.354 & 1.250 & 1.458 & 25.8 & .000 \\
\hline$\beta_{2}$ & -0.152 & -0.165 & -0.139 & -23.0 & .000 \\
\hline
\end{tabular}




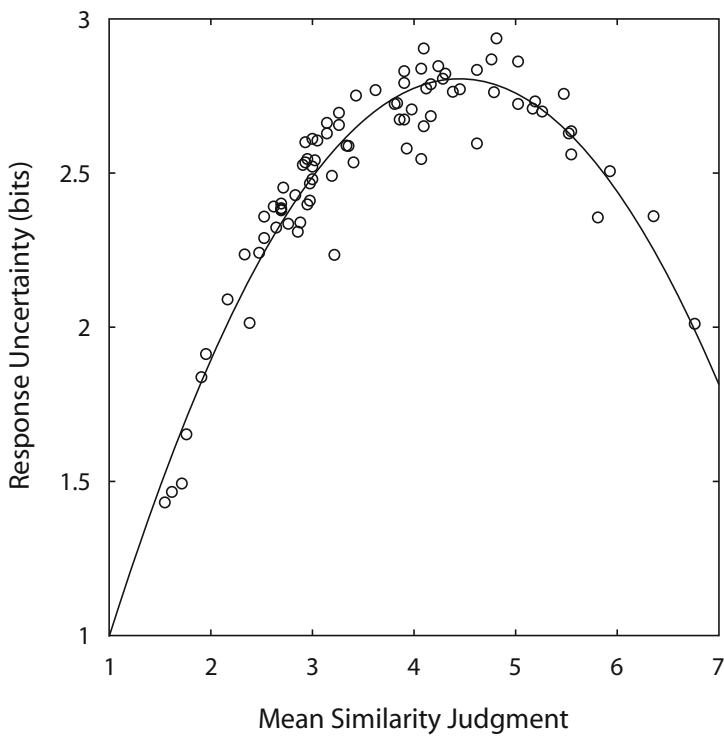

Figure 6. Response uncertainty as a function of mean similarity judgment for 84 stimulus pairs. Each circle represents a pair of facial expressions. The solid line represents the best-fitting quadratic equation.

The hypothesized inverted- $U$ effects were found relating mean RT and mean similarity judgment, as well as relating response uncertainty and mean similarity judgment. The difference in mean RT between the maximum and minimum values of the fitted quadratic equation is about $600 \mathrm{msec}$, much higher than $70 \mathrm{msec}$ (the widest systematic error likely caused by digit naming times). Therefore, we are confident that the inverted-U RT effect that we found cannot be attributed to an artifact involving number naming times.

\section{EXPERIMENT 2 Judgments of Gender and Maturity}

To make the argument for the generality of inverted-U effects more compelling, we had other participants perform two other tasks, and tested the presence of these effects on each task. We chose tasks in the field of face perception and selected the perception of femininity/masculinity and the perception of facial maturity. Gender is a very important property of faces. Indeed, when performing a principal component analysis of facial images, the first eigenvector explaining most of the variance is linked to gender (Valentin, Abdi, Edelman, \& O'Toole, 1997). Facial maturity has been shown to have a major impact on perceivers. It has been linked to attractiveness, credibility, and the severity of sentences pronounced against defendants (for a review, see Zebrowitz, 1997). The choice of the gender and maturity scales also stems from unpublished pilot studies performed in our laboratory demonstrating the high test-retest reliability of these measures. Furthermore, several other researchers have validated these measures (McArthur \& Berry, 1987; O'Toole et al., 1998; Zebrowitz \& Montepare, 1992).

\section{Method}

Participants. Participants were $36 \mathrm{McGill}$ University psychology students (13 men) and ranged in age from 18 to 44 years. The mean age was 21.9 years, with a standard deviation of 5.1 years. Participants were paid Can $\$ 10$ for their participation. All participants reported normal or corrected-to-normal vision.

Stimuli. Stimuli were taken from the Max Planck Institute Face Database, which was provided by the Max Planck Institute for Biological Cybernetics in Tübingen, Germany (Blanz \& Vetter, 1999; Troje \& Bülthoff, 1996). The stimuli in this database are 3-D models of faces. Each facial model is made of two files: a texture file and a 3 -D coordinate mesh. We accomplished the visual rendering process using a MATLAB 5.1 program on a Macintosh G4 computer. To generate the 2-D projections of the 3-D stimuli, we had to select a number of options. We chose to have perspective in the projection; to put the virtual camera at $5 \mathrm{ft}$ from the virtual faces and at the height of the eyes; to put the virtual light at $3 \mathrm{ft}$; to set the light angle at $45^{\circ}$; and to have the background black. The ambient lighting strength was set to .8 , the diffuse strength to .5 , the specular strength to .1 , and the specular exponent to 10 . We used a total of 100 stimuli (50 male and 50 female stimuli). For the 10 additional practice trials, we used different posers from the same database. Hair had already been removed from the original images. Each image was normalized in order to put the middle of the eyes at the same height, and intereye distance was standardized. This operation was achieved by manually setting reference points on each face and by automatically translating, cropping, and resizing each image with MATLAB programs. Images were presented in color. Examples of the stimuli are provided in Figure 7.

Apparatus. The experiment was programmed in MATLAB 5.1, using the Psychophysics Toolbox (Brainard, 1997), and executed on a Macintosh G3 computer. Participants sat at about $60 \mathrm{~cm}$ from a 48-cm Macintosh ColorSync monitor. Each stimulus presentation was synchronized with the screen refresh. This setup provides a 16-msec precision in the measurement of RT (Brainard, Pelli, \& Ingling, 2002)

Procedure. The experiment included two tasks, a gender and a maturity task. Participants were randomly divided into two groups. There were 18 participants in each group, with 7 men in Group 1 and 6 in Group 2. The order of presentation of the two tasks was counterbalanced between the two groups. Group 1 performed the femininity task first, whereas Group 2 performed the maturity task
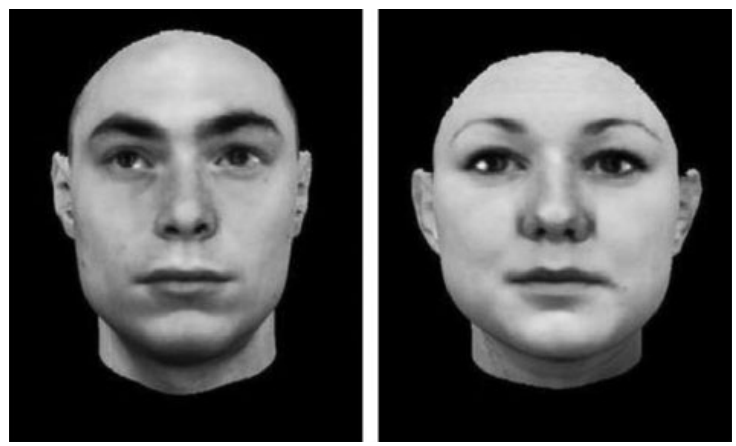

Figure 7. Examples of a male and a female stimulus used in the maturity and gender tasks. Note that participants saw the stimuli in color. From the Max Planck Institute Face Database. Reproduced with the permission of the Max Planck Institute for Biological Cybernetics, Tübingen, Germany. 
first. Each task was structured in the same way, with the instructions at the beginning, 10 practice trials, a block of 50 trials, a break, and the last block of 50 trials.

The experimenter trained participants on the method of answering. In order to accurately measure RT, participants had to press the "v" key to begin each trial. They then had to leave their finger above the " $v$ " key until they made their judgment, which they recorded by pressing the appropriate number key, 1 through 9 (at the top of the QWERTY keyboard). They were told to use the same index finger in pressing the " $\mathrm{v}$ " key and the selected number key.

Each trial was structured the same way. A fixation cross was presented for $250 \mathrm{msec}$, followed by a blank screen for $200 \mathrm{msec}$, and finally by the facial image. The image appeared until the participant typed in a response or until $6,000 \mathrm{msec}$ had elapsed. The relevant question (given below) was displayed at the bottom of the screen at the same time as the image. Average face width sustained an angle of $8.1^{\circ}$. Male and female images were mixed. The order of presentation of the stimuli was randomized across participants. There was an intertrial interval of $1,000 \mathrm{msec}$, after which participants had to press a key to start the next trial.

In the gender task, participants answered the question "How masculine or feminine does this face look in this picture?" using a rating scale from 1 (highly masculine) to 9 (highly feminine). These endpoint labels have been used in other studies (e.g., Dunkle \& Francis, 1990). In the maturity task, participants answered the question "How mature does this face look in this picture?" using a rating scale from 1 (baby faced) to 9 (mature faced). These end-point labels have also been used in other studies (e.g., Copley \& Brownlow, 1995).

\section{Results}

Validity tests. We performed several tests to assess the validity of the results. We correlated individual participants' gender and maturity scores with the mean scores, which revealed that the responses of 1 participant in the gender task correlated negatively with mean scores in that task, so the data of this outlier were not analyzed. No outlier was identified in the maturity task. Data from the two groups were analyzed together to control for any potential order effect. We computed the mean RT for each participant and each task. Each of them had a mean RT within 2.44 standard deviations of the grand mean RT across participants (i.e., $p>.01$ ). The split-sample correlation technique was used to assess the test-retest reliability of measurements. As in Experiment 1, we repeated this operation 10 times and averaged the results. For the gender task, the reliability estimates (computed with the Spearman-Brown correction formula) for mean RT, response uncertainty, and average judged femininity score were $.818, .877$, and .992 , respectively. For the maturity task, these reliability estimates for mean RT, response uncertainty, and average judged maturity score were .652 , .725 , and .977 , respectively. Finally, we computed the skew of mean RT measures. We found that the skew was low enough to assume symmetry and performed regressions using mean RT measures untransformed $\left(\gamma_{1}=-0.0134\right.$ and -0.115 , for gender and maturity, respectively).

Inverted-U effects. Figure 8 plots mean RT as a function of average judged femininity. On this figure and the three following ones, stimuli are represented by the letters $\mathrm{M}$ and $\mathrm{F}$, which stand for male and female faces, respectively. Regression analyses were performed on mean $\mathrm{RT}$ and average judged femininity. The best-fitting linear equation is significant $\left(R^{2}=.0787\right)[F(1,98)=8.37, p<$

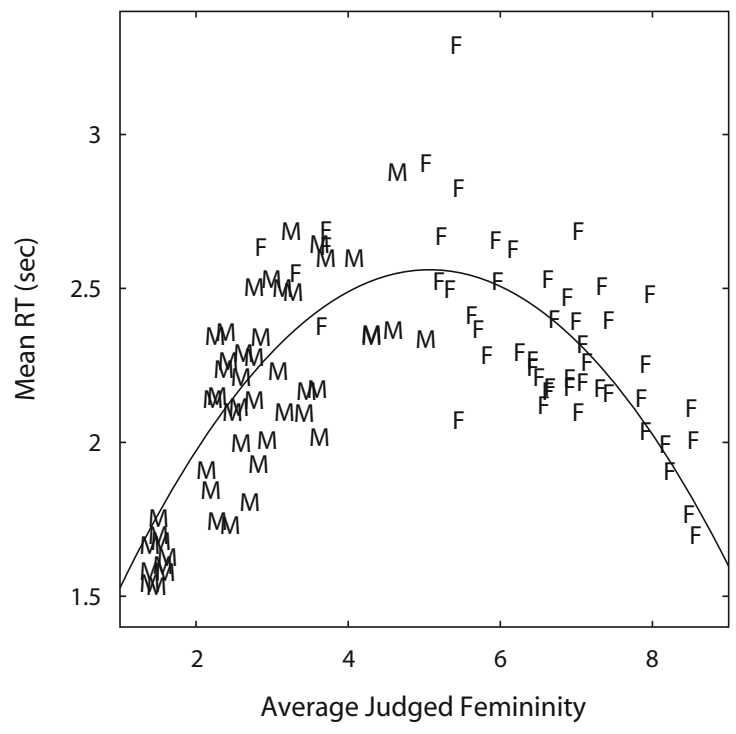

Figure 8. Mean response time (RT) as a function of average judged femininity for 100 stimuli. The letters $M$ and $F$ represent male and female faces, respectively. The solid line represents the best-fitting quadratic equation.

$.01]$. The best-fitting quadratic equation is also significant $\left(R^{2}=.592\right)[F(2,97)=70.5, p<.001]$. A partial $F$ test reveals that the quadratic equation is significantly superior to the linear one $[F(1,97)=122.27, p<.001]$. The detailed results for each parameter of the linear and the quadratic equation are provided in Table 2. We looked at individual RT data by computing mean RT for three sections of the response scale ( 1 to 3,4 to 6 , and 7 to 9 ) for each participant. We found that 26 out of 30 participants (i.e., $87 \%$ ) showed the inverted-U RT effect (5 were excluded because they did not give any answer in one of the three sections of the scale). We found that averaging across the 4 participants who did not individually show an inverted-U RT effect produced an inverted-U mean RT effect. The plot of response uncertainty as a function of average judged femininity is shown in Figure 9. The best-fitting linear equation is significant $\left(R^{2}=.103\right)$ $[F(1,98)=11.28, p<.005]$. The best-fitting quadratic equation is also significant $\left(R^{2}=.898\right)[F(2,97)=426.3$, $p<.001]$. A partial $F$ test reveals that the quadratic equation is significantly superior to the linear one $[F(1,97)=$ $754.53, p<.001]$. The detailed results for each parameter of the linear and the quadratic equation are provided in Table 2.

Figure 10 plots mean RT as a function of average judged maturity scores. A regression analysis was performed. The best-fitting linear equation is not significant $\left(R^{2}=.003\right)[F(1,98)=0.293, p>.25]$. However, the best-fitting quadratic equation is significant $\left(R^{2}=\right.$ .458) $[F(2,97)=41.0, p<.001]$. A partial $F$ test reveals that the quadratic equation is significantly superior to the linear one $[F(1,97)=81.50, p<.001]$. The detailed results for each parameter of the linear and the quadratic 
Table 2

Results of Linear and Quadratic Regressions for

(A) Response Time and (B) Response Uncertainty, As a Function of Average Judged Femininity

\begin{tabular}{llllll}
\hline \multirow{4}{*}{ Parameter } & Coefficient & Lower & Upper & & \\
& Bound & Bound & $t$ & Significance \\
\hline
\end{tabular}

(A) Response Time As a Function of Average Judged Femininity Linear Model

\begin{tabular}{|c|c|c|c|c|c|}
\hline$\beta_{0}$ & 2.040 & 1.896 & 2.189 & 27.6 & .000 \\
\hline$\beta_{1}$ & 0.0426 & 0.0130 & 0.0720 & 2.8 & .005 \\
\hline \multicolumn{6}{|c|}{ Quadratic Model } \\
\hline$\beta_{0}$ & 0.957 & 0.738 & 1.175 & 8.7 & .000 \\
\hline$\beta_{1}$ & 0.633 & 0.525 & 0.740 & 11.6 & .000 \\
\hline 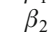 & -0.0624 & -0.074 & -0.051 & -11.0 & 00 \\
\hline
\end{tabular}

(B) Response Uncertainty As a Function of Average Judged Femininity Linear Model

\begin{tabular}{rrrrrr}
$\beta_{0}$ & 1.798 & 1.597 & 1.999 & 17.7 & .000 \\
$\beta_{1}$ & 0.0677 & 0.028 & 0.108 & 3.3 & .001 \\
Quadratic Model & & & & \\
$\beta_{0}$ & -0.0787 & -0.230 & 0.073 & -1.0 & .306 \\
$\beta_{1}$ & 1.088 & 1.013 & 1.163 & 28.8 & .000 \\
$\beta_{2}$ & -0.108 & -0.116 & -0.100 & -27.4 & .000 \\
\hline
\end{tabular}

equation are provided in Table 3 . We computed the mean RT for responses on three sections of the response scale for each participant. We found that 24 out of 26 participants (i.e., 92\%) showed the inverted-U RT effect ( 9 participants were excluded because they did not give any answer in one of the three sections of the range). Averaging across the only 2 participants who did not show the inverted-U RT effect did not produce an inverted-U mean RT effect. The plot of response uncertainty as a function of average judged maturity judgments is shown in Figure 11. The best-fitting linear equation is significant $\left(R^{2}=.0566\right)[F(1,98)=5.88, p<.025]$. The bestfitting quadratic equation is also significant $\left(R^{2}=.718\right)$ $[F(2,97)=123.3, p<.001]$. A partial $F$ test reveals that the quadratic equation is superior to the linear one $[F(1,97)=227.16, p<.001]$. The detailed results for each parameter of the linear and the quadratic equation are provided in Table 3 .

\section{Discussion}

The first goal was to test the hypothesis that there is an inverted-U relation linking response uncertainty with average scores and linking mean RT with average scores. This goal was achieved for the Likert-type scales (of one item) of femininity and maturity. However, the fit was better for the femininity task than for the maturity task. It appears that the maturity task is noisier, or more difficult for participants, than the gender task. This is confirmed by the fact that the split-sample correlations were lower for the maturity scores than for the femininity scores.

Although we used a normal keyboard with an accuracy of only $16 \mathrm{msec}$ for the measurement of RT, we still obtained a highly significant effect (i.e., $p<.001$ ) for both the femininity and the maturity task. Furthermore, the inverted-U RT effect cannot be attributed to an artifact of the response method because on a QWERTY keyboard, the distance is greater between the initial position of the finger (the "v" key) and the extremities (keys " 1 " or "9") than between the " $v$ " key and the center of the response range (key " 5 "). The bias of a normal keyboard should have produced a U-shaped RT effect, which is the opposite of what we found. Therefore, the use of a normal keyboard gives even more support to our results.

In Experiments 1 and 2, the inverted- $U$ relation found for response uncertainty was less noisy than the relation found with mean RT. Mean RT is probably influenced by the sources of noise involved in response production (e.g., variations in the delay for the finger to reach a key),

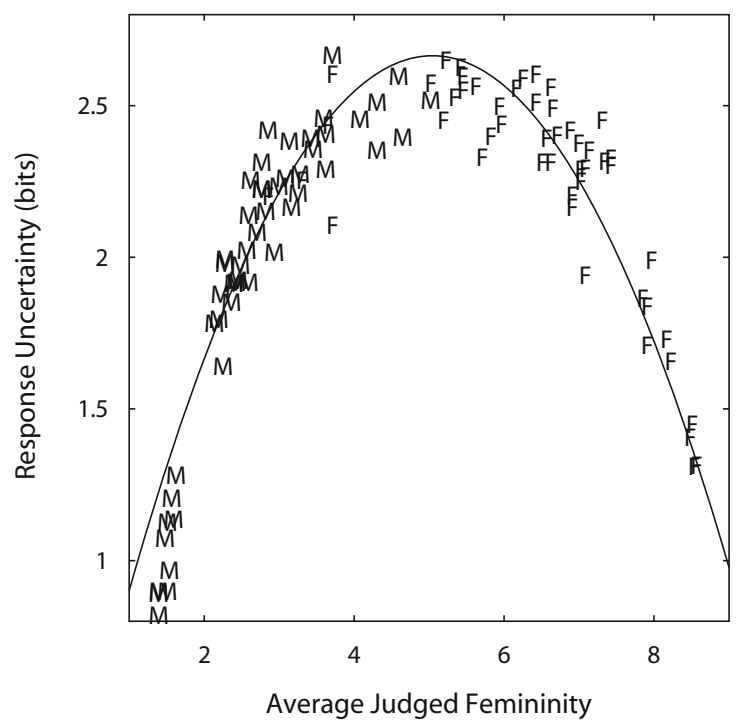

Figure 9. Response uncertainty as a function of average judged femininity for 100 stimuli. The letters $M$ and $F$ represent male and female faces, respectively. The solid line represents the bestfitting quadratic equation. 


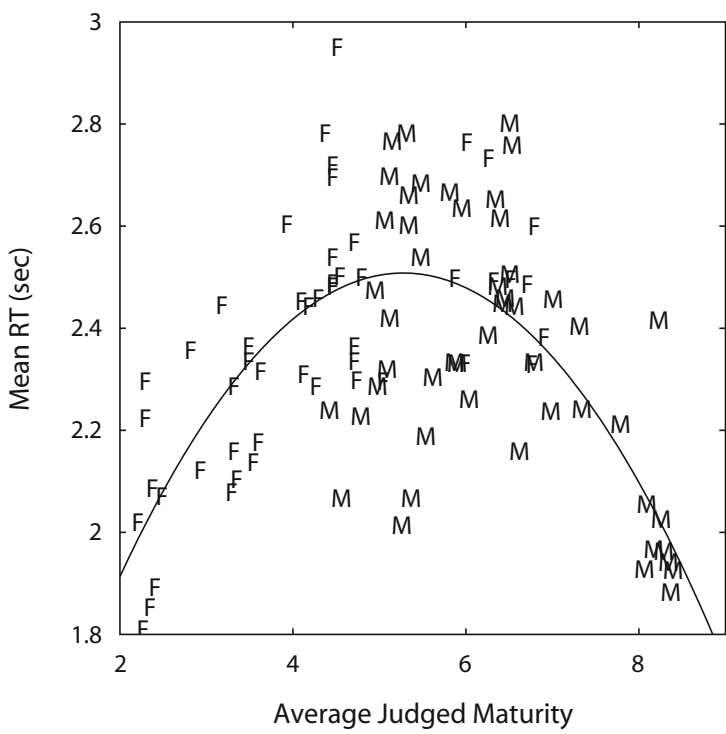

Figure 10. Mean response time (RT) as a function of average judged maturity for 100 stimuli. The letters $M$ and $F$ represent male and female faces, respectively. The solid line represents the best-fitting quadratic equation.

whereas response uncertainty should be less influenced by this source of variation (except when the finger accidentally hits the wrong key, changing response probabilities and response uncertainty). This suggests that there probably are experimental designs in which response uncertainty would be a more reliable measure than mean RT. Also, because response uncertainty summarizes the data for all response probabilities for a given stimulus, it may also be a more useful (and reliable) measure than probability correct, which is used in many tasks, such as in absolute identification.

\section{GENERAL DISCUSSION}

The main goal of this article is to propose that the inverted-U effects found in absolute identification and in Likert-type scales of personality and attitude questionnaires are part of a more general phenomenon. We hypothesized that when judging stimuli on a scale of polytomous ordered categories, the task constraints force the use of common mental processes (the stimulus representation and response selection phases) that give rise to these effects. Therefore, we predicted that the effects should also occur in a very different type of task sharing the same constraints - in this case, the judgment of subjective properties of faces on a Likert-type scale.

Our results in three tasks are consistent with this hypothesis. The inverted- $U$ response uncertainty effect was striking, as shown by the $R^{2}$ for the quadratic fit of similarity, gender, and maturity tasks: $.912, .898$, and .718, respectively. The inverted-U RT effect that we found was also strong in that the correlation coefficients were relatively high, with $R^{2}$ equal to $.528, .592$, and .458 for the similarity, gender, and maturity tasks, respectively. We also found that the six quadratic equations fitting these effects were all significantly superior to the linear equation at $p<.001$. The six quadratic parameters $\beta_{2}$ were also all significant at $p<.001$ (see Tables 1,2, and 3). We also found an inverted-U RT effect in individual results for $61 \%, 87 \%$, and $92 \%$ of the participants for the similarity, gender, and maturity tasks, respectively. Furthermore, when we averaged responses of the individual participants who did not show the inverted-U RT effect, we obtained an inverted-U mean RT effect for the similarity and gender tasks but not for the maturity task, in which there were only 2 such participants. These results suggest that participants who did not show an inverted-U RT effect simply showed more noise in their response patterns. Therefore,

Table 3

Results of Linear and Quadratic Regressions for

(A) Response Time and (B) Response Uncertainty, As a Function of Average Judged Maturity

\begin{tabular}{llllll}
\hline \multirow{2}{*}{ Parameter } & Coefficient & Lower & Upper & & \\
& Bound & Bound & $t$ & Significance \\
\hline
\end{tabular}

(A) Response Time As a Function of Average Judged Maturity

Linear Model

\begin{tabular}{|c|c|c|c|c|c|}
\hline$\beta_{0}$ & 2.319 & 2.152 & 2.487 & 27.4 & .000 \\
\hline$\beta_{1}$ & 0.00842 & -0.022 & 0.039 & 0.54 & .589 \\
\hline \multicolumn{6}{|c|}{ Quadratic Model } \\
\hline$\beta_{0}$ & 0.966 & 0.644 & 1.288 & 5.9 & .000 \\
\hline$\beta_{1}$ & 0.584 & 0.456 & 0.713 & 9.0 & .000 \\
\hline$\beta_{2}$ & -0.0553 & -0.068 & -0.043 & -9.0 & .000 \\
\hline
\end{tabular}

(B) Response Uncertainty As a Function of Average Judged Maturity Linear Model

\begin{tabular}{|c|c|c|c|c|c|}
\hline$\beta_{0}$ & 2.699 & 2.492 & 2.907 & 25.7 & .000 \\
\hline$\beta_{1}$ & -0.0467 & -0.085 & -0.008 & -2.4 & .017 \\
\hline \multicolumn{6}{|c|}{ Quadratic Model } \\
\hline$\beta_{0}$ & 0.621 & 0.324 & 0.917 & 4.1 & .000 \\
\hline$\beta_{1}$ & 0.838 & 0.719 & 0.956 & 14.0 & .000 \\
\hline$\beta_{2}$ & -0.0850 & -0.096 & -0.074 & -15.0 & .000 \\
\hline
\end{tabular}




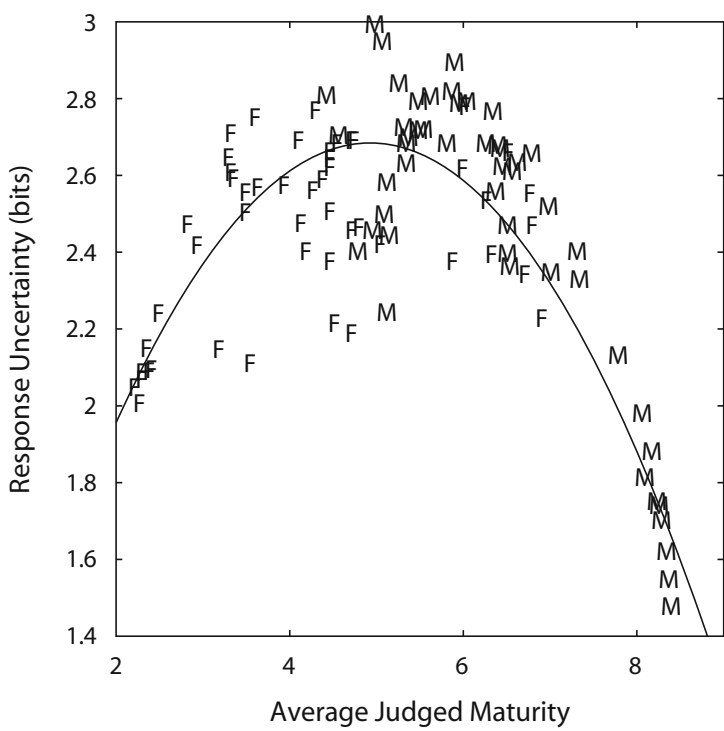

Figure 11. Response uncertainty as a function of average judged maturity for 100 stimuli. The letters $M$ and $F$ represent male and female faces, respectively. The solid line represents the best-fitting quadratic equation.

from many angles of analysis, the inverted-U effects were strong and robust.

These results may be interpreted using the classic phases of decision making (i.e., stimulus analysis, stimulus representation, response selection, and response production). The fact that we found inverted-U effects to be independent of the response mode (keypress or voice key) supports our hypothesis that they do not occur in the response production phase. This is similar to the findings of Lacouture and Marley (2004) in absolute identification. The existence of the inverted- $U$ effect over a diversity of stimuli and tasks (in absolute identification, our psychophysical tasks using social stimuli, and psychometrics) also supports our hypothesis that they do not occur in the stimulus analysis phase of the decision process. This finding is parallel to the finding in absolute identification that the inverted-U RT effect is independent of the dimensionality of the stimuli (Monahan \& Lockhead, 1977). Therefore, we conclude that the inverted-U effects occur in the stimulus representation or response selection phase. We think that, in the second phase, stimuli are mapped to one dimension because that is the dimensionality of the response on the Likert-type item; that is, the task forces this representation. As discussed earlier, this assumption is common to many models of absolute identification (e.g., Brown et al., 2008).

Our explanation of the inverted- $U$ effects is at odds with the explanations proposed by self-schema researchers, who usually attribute the inverted-U RT effect to the Kuiper and Rogers (1979) two-process interpretation or Kuiper's (1981) prototype hypothesis, which involve the self-schema (Akrami et al., 2007; Casey \& Tryon, 2001; Catanzaro, 1997; Crandall, 1998). These researchers did not make the connection with absolute identification. Rather than adopting this domain-specific explanation, we think that the inverted-U RT effect may simply be related to "stimulus contents ambiguity or uncertainty" (see Casey \& Tryon, 2001, for similar views). Of course, our experiment was not designed to test the self-schema interpretations of the inverted-U RT effect. However, our results should lead selfschema researchers to reexamine the necessity of a twoprocess mechanism in the judgment of items on a Likerttype scale. It seems that the most parsimonious explanation of the inverted-U effects lies in the stimulus representation and response selection phases in which limited human attention capacity and noise dynamics play a key role.

Our results could have applications for self-schema researchers (e.g., Andrews, 2003), for researchers studying multidimensional spaces derived from similarity measures (e.g., Spencer-Smith et al., 2001), as well as for researchers studying the perception of gender and facial maturity (e.g., O'Toole et al., 1998; Zebrowitz \& Montepare, 1992). We also think that our findings will further generalize to other judgments of subjective properties commonly used in face perception, such as the judgment of dominance (e.g., Mignault \& Chaudhuri, 2003), emotional intensity (e.g., Atkinson, Dittrich, Gemmell, \& Young, 2004), grotesqueness (e.g., Bartlett \& Searcy, 1993), attractiveness, familiarity, likableness, and typicality (e.g., Vokey \& Read, 1992). Inverted-U RT and response uncertainty effects may even generalize to most judgments using Likerttype scales. For example, an inverted-U RT effect has been found in metacognition for confidence judgments (Baranski \& Petrusic, 2001). Before proposing other domainspecific explanations in this field or in other ones, it is important to realize that a single general phenomenon may be causing all of them.

In summary, this is the first time that the inverted-U effects have been studied in the judgment of subjective properties of stimuli on Likert-type scales and in face perception. These results extend the findings of inverted-U effects in absolute identification (e.g., Brown et al., 2008) and of an inverted-U RT effect in confidence judgments (Baranski \& Petrusic, 2001) and in personality and attitude questionnaires (e.g., Akrami et al., 2007). The existence of these effects in such diverse fields supports the hypothesis that they are caused by a general phenomenon. Furthermore, our explanation, as opposed to the two-process interpretation, offers a unifying framework for experiments in both psychophysics and psychometrics. Obviously, further research will be necessary to assess the universality of these inverted-U effects. In particular, future efforts should also be devoted to studying additional characteristics of the inverted-U effects (e.g., sequential effects), and then to developing a model of Likert-type scaling explaining these effects and their characteristics. The models already developed for absolute identification would seem to be a good starting point for such investigations.

\section{AUTHOR NOTE}

The research was supported by two fellowships to the first author, the first from the Fonds pour les Chercheurs et l'Aide à la Recherche 
(FCAR) and the second from the National Engineering and Research Council of Canada (NSERC); by NSERC Discovery Grant 8124-98 to A.A.J.M.; and by an NSERC Strategic Grant to A.C. The authors thank Janet Cleveland for her editorial assistance and the following research assistants for data collection and stimulus preparation. Experiment 1: Manjit-Kaur Singh, Cinderella Wingsze, Mélissa Martin, Erin Putterman, Lori-Ann Ciolek, Isabelle Brown Tesolin, Lisanne Grenier-Houde, Julie Canaan, and Jo-Ann Wilkins. Experiment 2: Farah Averill. Correspondence should be addressed to A. Mignault, Department of Psychology, McGill University, Montreal, QC, H3A 1B1 Canada (e-mail: alain@ego.psych.mcgill.ca).

\section{REFERENCES}

Akrami, N., Hedlund, L.-E., \& Ekehammar, B. (2007). Personality scale response latencies as self-schema indicators: The inverted-U effect revisited. Personality \& Individual Differences, 43, 611-618.

Alvarado, N. (1996). Congruence of meaning between facial expressions of emotion and selected emotion terms. Motivation \& Emotion, 20, 33-61.

ANDREWs, B. S. (2003). The role of response latency in differentiating between honest responders, educated dissimulators, and coachededucated dissimulators. Unpublished doctoral dissertation, Southern Illinois University.

Atkinson, A. P., Dittrich, W. H., Gemmell, A. J., \& Young, A. W. (2004). Emotion perception from dynamic and static body expressions in point-light and full-light displays. Perception, 33, 717-746.

Avant, L. L., Bevan, W., \& Wing, H. (1968). Effects of varying length of stimulus series and response scale upon response latency, response uncertainty, and transmitted information. Perception \& Psychophysics, 3, 449-452.

Baranski, J. V., \& Petrusic, W. M. (2001). Testing architectures of the decision-confidence relation. Canadian Journal of Experimental Psychology, 55, 195-206.

Bartlett, J. C., \& Searcy, J. (1993). Inversion and configuration of faces. Cognitive Psychology, 25, 281-316.

BEHAR, I. (1963). On the relation between response uncertainty and reaction time in category judgments. Perceptual \& Motor Skills, 16 595-596.

Blanz, V., \& VetTer, T. (1999). A morphable model for the synthesis of 3D faces. In Proceedings of the 26th Annual Conference on Computer Graphics and Interactive Techniques, SIGGRAPH '99 (pp. 187-194). New York: ACM Press/Addison-Wesley.

BraIDA, L. D., \& DurLaCh, N. I. (1972). Intensity perception: II. Resolution in one-interval paradigms. Journal of the Acoustical Society of America, 51, 483-502.

Brainard, D. H. (1997). The Psychophysics Toolbox. Spatial Vision, 10, 433-436.

Brainard, D. H., Pelli, D., \& Ingling, A. (2002). Psychophysics Toolbox questions. Retrieved January 19, 2007, from psychtoolbox.org/ PTB-2/questions.html\#reaction.

Brown, S. D., Marley, A. A. J., Donkin, C., \& Heathcote, A. (2008) An integrated model of choices and response times in absolute identification. Psychological Review, 115, 396-425.

CASEY, M. M., \& Tryon, W. W. (2001). Validating a double-press method for computer administration of personality inventory items. Psychological Assessment, 13, 521-530.

Catanzaro, J. M. (1997). The effect of response latencies on the validity of personality scales. Unpublished doctoral dissertation, Fordham University.

Coolican, H. (1994). Research methods and statistics in psychology (2nd ed.). London: Hodder \& Stoughton.

Copley, J. E., \& Brownlow, S. (1995). The interactive effects of facial maturity and name warmth on perceptions of job candidates. Basic \& Applied Social Psychology, 16, 251-265.

Costa, P. T., JR., \& McCrae, R. R. (1992). Normal personality assessment in clinical practice: The NEO Personality Inventory. Psychological Assessment, 4, 5-13.

Crandall, B. D. (1998). Item response latency in computerized personality assessment and the effect of socially desirable responding. Dissertation Abstracts International, 59(3-B), 1363. (UMI No. AAM9827505)
Damian, M. F. (2004). Asymmetries in the processing of Arabic digits and number words. Memory \& Cognition, 32, 164-171.

DUNKLE, J. H., \& FranCIS, P. L. (1990). The role of facial masculinity/ femininity in the attribution of homosexuality. Sex Roles, 23, 157-167.

Ekman, P., \& Friesen, W. V. (1976). Pictures of facial affect. Palo Alto, CA: Consulting Psychologists Press.

Green, D. M., \& Swets, J. A. (1966). Signal detection theory and psychophysics. New York: Wiley.

JuDD, C. M., \& KuLIK, J. A. (1980). Schematic effects of social attitudes on information processing and recall. Journal of Personality \& Social Psychology, 38, 569-578

KUIPER, N. A. (1981). Convergent evidence for the self as a prototype: The "inverted-U RT effect" for self and other judgments. Personality \& Social Psychology Bulletin, 7, 438-443.

KuIPER, N. A., \& Rogers, T. B. (1979). Encoding of personal information: Self-other differences. Journal of Personality \& Social Psychology, 37, 499-514.

LaCouture, Y., LI, S.-C., \& Marley, A. A. J. (1998). The roles of stimulus and response set size in the identification and categorisation of unidimensional stimuli. Australian Journal of Psychology, 50, 165-174

Lacouture, Y., \& Marley, A. A. J. (1995). A mapping model of bow effects in absolute identification. Journal of Mathematical Psychology, 39, 383-395.

Lacouture, Y., \& Marley, A. A. J. (2004). Choice and response time processes in the identification and categorization of unidimensional stimuli. Perception \& Psychophysics, 66, 1206-1226.

Lemay, G., Kirouac, G., \& Lacouture, Y. (1995). Expressions faciales émotionnelles spontanées dynamiques et statiques: Comparaison d'études de jugement catégoriel et dimensionnel [Dynamic and static spontaneous facial expressions of emotion: A comparison of categorical and dimensional judgments]. Canadian Journal of Behavioural Science, 27, 125-139.

Luce, R. D., \& Nosofsky, R. M. (1984). Attention, stimulus range, and identification of loudness. In S. Kornblum \& J. Requin (Eds.), Preparatory states and processes (pp. 3-25). Hillsdale, NJ: Erlbaum.

MARLEY, A. A. J., \& CoOK, V. T. (1984). A fixed rehearsal capacity interpretation of limits on absolute identification performance. British Journal of Mathematical \& Statistical Psychology, 37, 136-151.

McArthur, L. Z., \& BerRY, D. S. (1987). Cross-cultural agreement in perceptions of babyfaced adults. Journal of Cross-Cultural Psychology, 18, 165-192.

Mignault, A., \& Chaudhuri, A. (2003). The many faces of a neutral face: Head tilt and perception of dominance and emotion. Journal of Nonverbal Behavior, 27, 111-132.

Mignault, A., Etcoff, N. L., \& Pentland, A. (2004). Comparing different emotional spaces. In A. Kappas (Ed.), Proceedings of the XIth Conference of the International Society for Research on Emotions (pp. 320-324). Amsterdam: ISRE Publications. Retrieved April 16, 2008, from facpub.stjohns.edu/ booner/ISRE/ISRE_ Proceedings_2000.pdf.

Miller, G. A. (1956). The magical number seven, plus or minus two: Some limits on our capacity for processing information. Psychological Review, 63, 81-97.

MonaHAn, J. S., \& LocKHEAD, G. R. (1977). Identification of integral stimuli. Journal of Experimental Psychology: General, 106, 94-110.

Morey, L. C. (1991). Personality Assessment Inventory. Odessa, FL: Psychological Assessment Resources, Inc.

Mueller, J. H., Thompson, W. B., \& Dugan, K. (1986). Trait distinctiveness and accessibility in the self-schema. Personality \& Social Psychology Bulletin, 12, 81-89.

Nosofsky, R. M. (1997). An exemplar-based random-walk model of speeded categorization and absolute judgment. In A. A. J. Marley (Ed.), Choice, decision, and measurement: Essays in honor of R. Duncan Luce (pp. 347-365). Mahwah, NJ: Erlbaum.

Ogawa, T., \& SuzuKi, N. (2000). Emotion space as a predictor of binocular rivalry. Perceptual \& Motor Skills, 90, 291-298.

O’Toole, A. J., Deffenbacher, K. A., Valentin, D., McKee, K., HuFF, D., \& ABDI, H. (1998). The perception of face gender: The role of stimulus structure in recognition and classification. Memory \& Cognition, 26, 146-160.

RamsaY, J. O. (1997). Multiscale II manual. Unpublished manuscript, McGill University, Montreal. 
Rouder, J. N. (2001). Testing evidence accrual models by manipulating stimulus onset. Journal of Mathematical Psychology, 45, 334-354.

Russell, J. A., \& Bullock, M. (1986). On the dimensions preschoolers use to interpret facial expressions of emotion. Developmental Psychology, 22, 97-102.

SCHNEIDER, W. (1988). Micro Experimental Laboratory: An integrated system for IBM PC compatibles. Behavior Research Methods, Instruments, \& Computers, 20, 206-217.

Shannon, C. E., \& Weaver, W. (1964). The mathematical theory of communication. Urbana: University of Illinois Press.

ShePard, R. N. (1962). The analysis of proximities: Multidimensional scaling with an unknown distance function I. Psychometrika, 27, 125-140.

Shiffrin, R. M., \& Nosofsky, R. M. (1994). Seven plus or minus two: A commentary on capacity limitations. Psychological Review, 101, 357-361.

Snodgrass, J. G., \& Yuditsky, T. (1996). Naming times for the Snodgrass and Vanderwart pictures. Behavior Research Methods, Instruments, \& Computers, 28, 516-536.

Spencer-Smith, J., Wild, H., Innes-Ker, Å. H., Townsend, J., Duffy, C., Edwards, C., ET AL. (2001). Making faces: Creating three-dimensional parameterized models of facial expression. Behavior Research Methods, Instruments, \& Computers, 33, 115-123.

Stewart, N., Brown, G. D. A., \& Chater, N. (2005). Absolute identification by relative judgment. Psychological Review, 112, 881-911.

Stilson, D. W., HaAseth, K., Walsmith, C. R., Schneider, R. J., \&
Rogers, M. (1972). Marbe's law, stimulus expectancy, and response competition. American Journal of Psychology, 85, 549-567.

Torgerson, W. S. (1958). Theory and methods of scaling. New York: Wiley.

Troje, N., \& Bülthoff, H. H. (1996). Face recognition under varying poses: The role of texture and shape. Vision Research, 36, 1761-1771.

Valentin, D., Abdi, H., Edelman, B., \& O’Toole, A. J. (1997). Principal component and neural network analyses of face images: What can be generalized in gender classification? Journal of Mathematical Psychology, 41, 398-412.

VoKey, J. R., \& READ, J. D. (1992). Familiarity, memorability, and the effect of typicality on the recognition of faces. Memory \& Cognition, 20, 291-302.

WeEms, G. H., \& OnwuegbuzIE, A. J. (2001). The impact of midpoint responses and reverse coding on survey data. Measurement \& Evaluation in Counseling \& Development, 34, 166-176.

ZeBrowitz, L. A. (1997). Reading faces: Window to the soul? Boulder, $\mathrm{CO}$ : Westview.

Zebrowitz, L. A., \& Montepare, J. M. (1992). Impressions of babyfaced individuals across the life span. Developmental Psychology, 28, 1143-1152.

(Manuscript received July 19, 2007; revision accepted for publication April 7, 2008.) 